\title{
A Hamiltonian weak-wave model for shallow-water flow
}

\author{
CAROLine Nore \\ Laboratoire de Physique Statistique, Ecole Normale Supérieure, \\ 24, rue Lhomond, 75231 Paris 05 Cedex, France \\ and \\ THEODORE G. SHEPHERD \\ Department of Physics, University of Toronto, Toronto M5S 1A7 Canada
}

\begin{abstract}
A reduced dynamical model is derived which describes the interaction of weak inertiagravity waves with nonlinear vortical motion in the context of rotating shallow-water flow. The formal scaling assumptions are (i) that there is a separation in timescales between the vortical motion and the inertia-gravity waves, and (ii) that the divergence is weak compared to the vorticity. The model is Hamiltonian, and possesses conservation laws analogous to those in the shallow-water equations. Unlike the shallow-water equations, the energy invariant is quadratic. Nonlinear stability theorems are derived for this system, and its linear eigenvalue properties are investigated in the context of some simple basic flows.
\end{abstract}




\section{Introduction}

An important area of research in geophysical fluid dynamics is the development of simplified models. Simplified models are reduced models of the full hydrodynamical equations of motion that retain the essential physics of the problem under investigation, yet are reduced in complexity and are therefore more amenable to theoretical analysis.

A generic property of atmospheric and oceanic dynamics is the existence of nonlinear vortical motion, evolving on an advective timescale, together with relatively fast acoustic and gravity wave motion. While both kinds of motion can be identified observationally, it is generally the case that the vortical motion overwhelmingly dominates the fast wave motion for both meso-scale and large-scale dynamics. This fact has led to a class of simplified models known as balanced models, which filter the fast wave motion and describe only the nonlinear vortical motion. Examples of such models include the barotropic vorticity equation, the quasi-geostrophic equations, and the semi-geostrophic equations (see e.g. Pedlosky 1987).

Yet while the fast wave motion is observationally found to be relatively weak, it is nevertheless ubiquitous. Moreover, there are strong theoretical reasons for believing that balanced models cannot be exact (even in principle), and that some degree of fast motion is inevitable. There is, therefore, considerable interest in the problem of the interaction between (slow) nonlinear vortical motion and (fast) weak waves. Clearly it would be desirable to avoid dealing with the full primitive equations of motion in theoretical investigations of this nature. Yet simplified models describing this kind of interaction do not exist, except for very special cases.

In this paper we address this problem of constructing simplified models describing the interaction of nonlinear vortical motion with weak waves under a separation in timescales. A traditional approach is through perturbation methods, employing an asymptotic expansion with the timescale ratio between the wave and vortical motion (i.e. the Mach, Froude, or Rossby number) serving as the small expansion parameter. However, such methods are generally carried only to leading order. This is, perhaps, not surprising in light of the 
fact that the higher-order corrections in traditional expansions will generically exhibit unbounded secular growth in time (Warn et al. 1995). Yet to obtain non-trivial coupling between the wave and vortical motion, one has to go beyond leading order.

A rather different approach is to argue that one should maintain the fundamental dynamical properties of the original system, notably its conservation properties. The importance of maintaining conservation properties in dynamical simplifications has been long recognized (Lorenz 1960, Sadourny 1975). More recently, it has been realized (e.g. Salmon 1983) that the most efficient way to do this is through maintaining the Hamiltonian structure of the dynamics: the simplifications in question invariably affect the conservative part of the dynamics, which for nearly every model in geophysical fluid dynamics is Hamiltonian (see e.g. Benjamin 1984, Shepherd 1990).

Ideally one would like to be able to satisfy both requirements, Hamiltonian structure and formal accuracy. However, so far they have proved to be incompatible beyond leading order. Arguments can be mustered for one approach or the other. We do not wish to enter that debate here, but simply choose to follow the Hamiltonian approach, guided by scaling arguments, in what follows — recognizing its possible limitations.

The system we shall investigate is rotating shallow-water flow. This is chosen because it is arguably the simplest system in geophysical fluid dynamics that contains the essential physics of nonlinear vortical motion coupled to fast (in this case inertia-gravity) waves. Yet the approach may be expected to have implications for other systems having the same essential characteristics, such as vortex/acoustic-wave interactions in compressible flow. Farge \& Sadourny (1989) proposed a weak-wave model for shallow-water flow, but their derivation was rather $a d h o c$ and their system has a number of unsatisfactory features. We are aware of no other study along these lines.

The plan of the paper is as follows. In Section 2 the properties of the shallow-water equations are briefly reviewed. Our weak-wave model is derived in Section 3, in a way that guarantees its Hamiltonian character. We then describe some of its properties: symmetries and conservation laws (Section 4); pseudoenergy and pseudomomentum invariants and nonlinear stability (Section 5); rigorous upper bounds on gravity-wave emission from 
unstable parallel vortical shear flows (Section 6); linear eigenvalues and instability (Section 7). Some of the limitations of our approach are discussed in Section 8.

\section{The shallow-water system}

The shallow-water equations, describing the dynamics of a shallow, homogeneous, rotating fluid, are given by (e.g. Pedlosky 1987)

$$
\boldsymbol{v}_{t}+(\boldsymbol{v} \cdot \boldsymbol{\nabla}) \boldsymbol{v}+f \hat{\boldsymbol{z}} \times \boldsymbol{v}=-g \boldsymbol{\nabla} h, \quad h_{t}+\boldsymbol{\nabla} \cdot(h \boldsymbol{v})=0,
$$

where $\boldsymbol{v}(x, y, t)$ is the (horizontal) velocity, $h(x, y, t)$ the fluid depth, $g$ the (constant) gravitational acceleration, $f$ the (constant) Coriolis parameter, $\hat{z}$ the unit vertical vector, and $\nabla \equiv(\partial / \partial x, \partial / \partial y) \equiv\left(\partial_{x}, \partial_{y}\right)$. We assume either an unbounded domain with appropriate decay conditions at infinity, or a doubly-periodic domain, or a combination of the two (i.e. unbounded in one direction and periodic in the other). This system has the following integral invariants: energy $\mathcal{H}=\int \frac{1}{2}\left(h|\boldsymbol{v}|^{2}+g h^{2}\right) d \boldsymbol{x}$; zonal absolute momentum $\mathcal{M}=\int h(u-f y) d \boldsymbol{x}$, where $\boldsymbol{v} \equiv(u, v) ;$ angular momentum $\mathcal{J}=\int h\left[x v-y u+\frac{f}{2}\left(x^{2}+y^{2}\right)\right] d \boldsymbol{x} ;$ and a family of Casimirs $\mathcal{C}=\int h C(q) d \boldsymbol{x}$ where $C(\cdot)$ is an arbitrary function and $q=$ $[f+\hat{\boldsymbol{z}} \cdot(\boldsymbol{\nabla} \times \boldsymbol{v})] / h$ is the potential vorticity. The Casimir invariants are a consequence of the material conservation of potential vorticity, $q_{t}+\boldsymbol{v} \cdot \nabla q=0$, which follows from (2.1).

The system (2.1) can be written in the generic Hamiltonian form (e.g. Shepherd 1990)

$$
\sigma_{t}=J \frac{\delta \mathcal{H}}{\delta \sigma}
$$

where $\sigma=(\boldsymbol{v}, h)^{T}$ is the state vector of the dynamical variables, $\mathcal{H}$ is the Hamiltonian, and the cosymplectic form $J$ is given by

$$
J=\left(\begin{array}{ccc}
0 & q & -\partial_{x} \\
-q & 0 & -\partial_{y} \\
-\partial_{x} & -\partial_{y} & 0
\end{array}\right)
$$


If one linearizes the dynamics (2.1) about the rest state $\boldsymbol{v}=0, h=H=$ const., the dispersion relation is third order: one root has frequency $\omega=0$, corresponding to the vortical motion; and there is a pair of roots with frequency $\omega= \pm f \sqrt{1+g H \kappa^{2} / f^{2} L^{2}}$, where $L$ is a horizontal length scale and $\kappa$ the $O(1)$ dimensionless wavenumber, corresponding to inertia-gravity waves. Allowing weak nonlinearity, the vortical motion has a timescale $L / V$, where $V$ is a typical velocity. The ratio of the inertia-gravity wave timescale to the vortical timescale is thus $\epsilon \equiv V /|\omega| L=R B / \sqrt{1+B^{2}}$, where $R \equiv V / f L$ is the Rossby number and $B \equiv f L / \sqrt{g H}$. Evidently there is a separation in timescales when $\epsilon \ll 1$. If one further assumes that the timescale of the dynamics is advective (so that the gravity waves are filtered), one obtains balanced dynamics: quasi-geostrophic dynamics for $R \ll 1$ with $B$ arbitrary, and barotropic dynamics for $B \ll 1$ with $R=O(1)$ (or equivalently $F \ll 1$, where $F \equiv V / \sqrt{g H}$ is the Froude number).

It may be noted that the energy of the full shallow-water equations (2.1) is cubic in the dependent variables, which is a consequence of the fact that the flow is divergent. (The same situation arises with compressible flow.) This presents some serious difficulties with respect to theoretical investigations. First, the partitioning of energy between different scales of motion is ambiguous, which is a problem for studies of turbulence: Warn (1986), Farge \& Sadourny (1989), and Yuan \& Hamilton (1994) have all had to invoke the approximation of a quadratic energy in their analyses of shallow-water turbulence, which is essentially a weak-wave approximation. Second, stability theorems based on the pseudomomentum and pseudoenergy, while easily derived for small-amplitude disturbances (Ripa 1983), do not appear to extend to finite amplitude (Shepherd 1992).

What we seek, therefore, is a weak-wave model for shallow-water flow that includes the following features: quadratic energy; Hamiltonian structure; appropriate conservation laws; quasi-geostrophic or barotropic vortical motion; inertia-gravity waves; and non-trivial coupling between the vortical motion and the inertia-gravity waves.

\section{A Hamiltonian weak-wave model}

We begin by transforming the dependent variables in a way that makes the vortical and wave motion more transparent in the Hamiltonian structure. In particular, choose 
$\tilde{q}=\hat{\boldsymbol{z}} \cdot(\boldsymbol{\nabla} \times \boldsymbol{v})-f \eta / H, \Delta=\boldsymbol{\nabla} \cdot \boldsymbol{v}$, and $\eta=h-H$ as the new dependent variables. Writing $\boldsymbol{v}=\hat{\boldsymbol{z}} \times \boldsymbol{\nabla} \psi+\nabla \chi$, we now non-dimensionalize the variables according to $\psi=V L \psi_{*}, \chi=$ $V L \chi_{*}, \tilde{q}=(V / L) \tilde{q}_{*}, \nabla=(1 / L) \nabla_{*}, t=(b / f) t_{*}$, and $\eta=H R b \eta_{*}$, where $b \equiv B / \sqrt{1+B^{2}}$. Note that $\tilde{q}_{*}=\nabla^{2} \psi_{*}-b \eta_{*}, \epsilon=R b$, and $b \leq 1$. Dropping the asterisks from now on, the resulting system can again be cast in the form $(2.2)$ with the state vector $\sigma=(\tilde{q}, \Delta, \eta)^{T}$, with $J$ given by

$$
J=\left(\begin{array}{ccc}
-\epsilon \partial\left(\frac{\tilde{q}}{1+\epsilon \eta},(\cdot)\right) & \epsilon \boldsymbol{\nabla} \cdot\left(\frac{\tilde{q}}{1+\epsilon \eta} \nabla(\cdot)\right) & 0 \\
-\epsilon \boldsymbol{\nabla} \cdot\left(\frac{\tilde{q}}{1+\epsilon \eta} \nabla(\cdot)\right) & -\epsilon \partial\left(\frac{\tilde{q}}{1+\epsilon \eta},(\cdot)\right) & -\nabla^{2}(\cdot) \\
0 & \nabla^{2}(\cdot) & 0
\end{array}\right)
$$

where $\partial(f, g) \equiv f_{x} g_{y}-f_{y} g_{x}$, and with the Hamiltonian

$$
\mathcal{H}=\int \frac{1}{2}\left\{(1+\epsilon \eta)\left(|\nabla \psi|^{2}+|\nabla \chi|^{2}\right)+2 \epsilon \eta \hat{\boldsymbol{z}} \cdot \nabla \psi \times \nabla \chi+\frac{\eta^{2}}{1+B^{2}}\right\} d \boldsymbol{x} .
$$

(Note that in obtaining (3.2), the Casimir invariant $\int\left(\frac{1}{2} g H^{2}+g H \eta\right) d \boldsymbol{x}$ has been removed from the original Hamiltonian.) To this point no approximation has been made.

We now invoke the assumption of a timescale separation, $\epsilon \ll 1$. To leading order, we simply take $\epsilon=0$ which yields

$$
\left.J=\left(\begin{array}{ccc}
0 & 0 & 0 \\
0 & 0 & -\nabla^{2}(\cdot) \\
0 & \nabla^{2}(\cdot) & 0
\end{array}\right), \quad \mathcal{H}=\int \frac{1}{2}\left\{|\nabla \psi|^{2}+|\nabla \chi|^{2}\right)+\frac{\eta^{2}}{1+B^{2}}\right\} d x
$$

with

$$
\frac{\delta \mathcal{H}}{\delta \tilde{q}}=-\psi, \quad \frac{\delta \mathcal{H}}{\delta \Delta}=-\chi, \quad \frac{\delta \mathcal{H}}{\delta \eta}=\frac{\eta}{1+B^{2}}-b \psi
$$

The system (3.3) describes the linearized dynamics

$$
\frac{\partial \tilde{q}}{\partial t}=0, \quad \frac{\partial \Delta}{\partial t}=b \nabla^{2} \psi-\frac{\nabla^{2} \eta}{1+B^{2}}, \quad \frac{\partial \eta}{\partial t}=-\Delta
$$


with no vortical evolution. We now ask: what is the simplest modification that one can make to (3.3) to include the nonlinear vortical dynamics, while retaining the Hamiltonian structure? Keeping all the $O(\epsilon)$ terms in (3.1) is not an option, because Jacobi's identity would then not hold: this is a typical problem in non-canonical Hamiltonian perturbation expansions. However, we may now invoke our second scaling assumption, namely that the divergence is weak compared with the vorticity: $|\chi| \ll|\psi|$. Note that the first column of (3.1) acts on $\delta \mathcal{H} / \delta \tilde{q}$, which to leading order [see (3.3c)] is given by $-\psi$; while the second column of $(3.1)$ acts on $\delta \mathcal{H} / \delta \Delta$, which to leading order [see (3.3c)] is given by $-\chi$. Thus we keep the dominant term in each of the three equations by adding the leading-order part of the upper-left entry of (3.1) to (3.3a), namely

$$
J=\left(\begin{array}{ccc}
-\epsilon \partial(\tilde{q},(\cdot)) & 0 & 0 \\
0 & 0 & -\nabla^{2}(\cdot) \\
0 & \nabla^{2}(\cdot) & 0
\end{array}\right)
$$

while keeping the quadratic Hamiltonian (3.3b). It may be verified that (3.5) satisfies all required properties of cosymplectic forms, including Jacobi's identity. Note, however, that although the gravity-wave motion must be weak, it cannot be too weak or the divergence equation will be badly approximated by the above procedure. In particular, in the divergence equation the term arising from the first column of (3.1) must be negligible against that arising from the third column; using the fact that the dimensionless gravity-wave frequency and $\tilde{q}$ are both $O(1)$, this requires $\epsilon|\psi| \ll|\chi|$. The formal scaling regime for the validity of this system is therefore:

$$
\epsilon \ll 1, \quad \epsilon|\psi| \ll|\chi| \ll|\psi| \text {. }
$$

If we absorb the factor $\epsilon$ in the nonlinear term by rescaling all the variables by $1 / \epsilon$, then $(3.3 \mathrm{~b}, \mathrm{c})$ and $(3.5)$ yields the system

$$
\frac{\partial \tilde{q}}{\partial t}=-\partial(\psi, \tilde{q}), \quad \frac{\partial \Delta}{\partial t}=\left(\frac{B^{2}-\nabla^{2}}{1+B^{2}}\right) \eta+b \tilde{q}, \quad \frac{\partial \eta}{\partial t}=-\Delta
$$


This, together with the definition $\tilde{q}=\nabla^{2} \psi-b \eta$, is our weak-wave model.

The system (3.7) contains both nonlinear vortical dynamics [through (3.7a)] and inertia-gravity wave dynamics [through $(3.7 \mathrm{~b}, \mathrm{c})]$. The coupling between the wave and vortical dynamics is seen to come through the linear forcing term $b \tilde{q}$ in the $\partial \Delta / \partial t$ equation, and through feedback from the wave dynamics onto the $\partial \tilde{q} / \partial t$ equation through $\psi$. The system is analogous to the reduced nine-component low-order model of Lorenz (1986, Eqn.(2)), which also has all the nonlinearity in the slow equation.

It is interesting to compare the system (3.7) with the weak-wave model of Farge \& Sadourny (1989, Eqn.(15)); the latter is given by

$$
\frac{\partial \tilde{q}}{\partial t}=-\partial(\psi, \tilde{q}), \quad \frac{\partial \Delta}{\partial t}=\left(\frac{B^{2}-\nabla^{2}}{1+B^{2}}\right) \eta+b \tilde{q}-\partial\left(\chi, \nabla^{2} \psi\right), \quad \frac{\partial \eta}{\partial t}=-\Delta-\partial(\psi, \eta) .
$$

Evidently (3.8) has additional nonlinear terms, but it is not clear how they arise from the original shallow-water system via (3.1). In addition, (3.8) does not appear to be Hamiltonian.

\section{Symmetries and conservation laws}

In this section we discuss some of the dynamical implications of the Hamiltonian structure of our weak-wave system. The reader may wish to consult Shepherd (1990) for further background on some of the points mentioned here.

The approximation leading to (3.7) consists of keeping the leading order (quadratic) approximation to the full Hamiltonian (3.2), together with the simplest approximation to the full cosymplectic form (3.1) that incorporates the vortical dynamics yet still satisfies the requisite properties, including Jacobi's identity. The resulting weak-wave system (3.7) is thereby Hamiltonian by construction. The cosymplectic form (3.5) represents the direct product of the non-canonical quasi-geostrophic $J$, namely $-\partial(\tilde{q},(\cdot))$, and the canonical linear-wave $J$. This kind of structure can be expected to be generic for geophysical fluid dynamical systems involving both fast and slow degrees of freedom.

Hamiltonian structure provides a clear connection between symmetries and conservation laws, via Noether's theorem, and allows for the identification of Casimir invariants. 
The Casimir invariants $\mathcal{C}$ are the solutions to $J(\delta \mathcal{C} / \delta \sigma)=0$; in the case of $(3.5)$, this condition leads to

$$
\frac{\delta \mathcal{C}}{\delta \tilde{q}}=\text { function of } \tilde{q}, \quad \frac{\delta \mathcal{C}}{\delta \Delta}=\text { const. }, \quad \frac{\delta \mathcal{C}}{\delta \eta}=\text { const. }
$$

which implies

$$
\mathcal{C}=\int\left\{C(\tilde{q})+c_{1} \Delta+c_{2} \eta\right\} d \boldsymbol{x}
$$

Here $C(\cdot)$ is an arbitrary function and $c_{1}, c_{2}$ are arbitrary constants. With the exception of the second term, which vanishes anyway, the Casimir invariants (4.2) are obvious analogues of those present in the original shallow-water system, except that there is no factor of $h$ in front of $C(\tilde{q})$ (this reflects the fact that the advection of $\tilde{q}$ in $(3.7 \mathrm{a})$ is solely by the non-divergent flow); also, $\int C(\tilde{q}) d \boldsymbol{x}$ is identical to the Casimir invariants found in quasigeostrophic dynamics.

Noether's theorem (see e.g. Benjamin 1984) states that if $\delta \sigma$ is an infinitesimal symmetry perturbation under which the Hamiltonian is invariant, then a functional $\mathcal{M}$ satisfying $\delta \sigma=J(\delta \mathcal{M} / \delta \sigma)$ is a conserved quantity. We consider both zonal translational symmetry and rotational symmetry. In the first case, this condition leads to

$$
-\partial\left(\tilde{q}, \frac{\delta \mathcal{M}}{\delta \tilde{q}}\right)=-\tilde{q}_{x}, \quad-\nabla^{2} \frac{\delta \mathcal{M}}{\delta \eta}=-\Delta_{x}, \quad \nabla^{2} \frac{\delta \mathcal{M}}{\delta \Delta}=-\eta_{x}
$$

which may be shown to imply (within a Casimir, of course) the zonal momentum invariant

$$
\mathcal{M}=\int\left(y \tilde{q}+\eta \frac{\partial \chi}{\partial x}\right) d \boldsymbol{x}
$$

In the case of rotational symmetry with respect to an angle $\theta$, Noether's theorem leads to the angular momentum invariant

$$
\mathcal{J}=\int\left(-\frac{1}{2} r^{2} \tilde{q}+\eta \frac{\partial \chi}{\partial \theta}\right) d \boldsymbol{x}
$$

The terms involving $\tilde{q}$ in (4.4) and (4.5) represent an approximation to the momentum and angular momentum of the rotational part of the flow, in vorticity form; they are identical 
to the momentum and angular momentum invariants of quasi-geostrophic dynamics. The terms involving $\chi$ in (4.4) and (4.5) represent the momentum and angular momentum of the divergent part of the flow.

\section{Pseudoenergy, pseudomomentum, and nonlinear stability}

A general property of Hamiltonian systems is that any basic state possessing a symmetry invariance is a constrained extremal of the integral invariant corresponding to that symmetry, the constraint being imposed through a suitable Casimir. This means that one may construct exact, finite-amplitude invariants that are quadratic to leading order in the disturbance to such basic states. For example, with steady basic states one can combine the energy and Casimir invariants to obtain a so-called pseudoenergy invariant. In the same way, with zonally symmetric basic states one can combine the zonal momentum and Casimir invariants to obtain a pseudomomentum invariant. Such invariants can be used to derive nonlinear stability theorems. See Shepherd $(1990,1994)$ for further background.

\section{(a) Zonally symmetric basic states}

We first consider disturbances $\left\{\tilde{q}^{\prime}, \Delta^{\prime}, \eta^{\prime}\right\}$ to a steady, zonally symmetric basic state $\left\{\tilde{q}_{0}(y), \Delta_{0}(y), \eta_{0}(y)\right\}$. In this case we can use the energy (3.3b), Casimir (4.2), and zonal momentum (4.4) invariants to construct the combined invariant

$$
\mathcal{A} \equiv(\mathcal{H}+\mathcal{C}+\alpha \mathcal{M})\left[\tilde{q}_{0}+\tilde{q}^{\prime}, \Delta_{0}+\Delta^{\prime}, \eta_{0}+\eta^{\prime}\right]-(\mathcal{H}+\mathcal{C}+\alpha \mathcal{M})\left[\tilde{q}_{0}, \Delta_{0}, \eta_{0}\right]
$$

where $\alpha$ is kept free. The Casimir $\mathcal{C}$ is determined by the extremal condition $\delta \mathcal{A}=0$, which leads to

$$
C^{\prime}\left(\tilde{q}_{0}\right)=\psi_{0}-\alpha y, \quad \Delta_{0}=0, \quad \eta_{0}=b\left(1+B^{2}\right) \psi_{0}
$$

(Note that $c_{1}$ and $c_{2}$ can be set to zero without loss of generality by imposing a suitable gauge condition on the potentials $\psi_{0}$ and $\chi_{0}$.) Conditions $(5.2 \mathrm{~b}, \mathrm{c})$ express geostrophic balance, which is required for a steady solution. Condition (5.2a) determines the functional dependence of $C(\cdot)$; thus the right-hand side of (5.2a) must be interpreted as a function of 
$\tilde{q}_{0}$. If we introduce the function $G_{\alpha}(\cdot)$ defined by $G_{\alpha}\left(\tilde{q}_{0}(y)\right)=\psi_{0}(y)-\alpha y$, then $\mathcal{A}$ can be written

$$
\begin{aligned}
\mathcal{A}=\int\left\{\frac{1}{2}\left(\left|\nabla \psi^{\prime}\right|^{2}+\left|\nabla \chi^{\prime}\right|^{2}+\frac{\left(\eta^{\prime}\right)^{2}}{1+B^{2}}\right)-\psi_{0} \tilde{q}^{\prime}+\int_{\tilde{q}_{0}}^{\tilde{q}_{0}+\tilde{q}^{\prime}} G_{\alpha}(\xi) d \xi+\alpha y \tilde{q}^{\prime}+\alpha \eta^{\prime} \frac{\partial \chi^{\prime}}{\partial x}\right\} d \boldsymbol{x} \\
=\int\left\{\frac{1}{2}\left(\left|\nabla \psi^{\prime}\right|^{2}+\left(\frac{\partial \chi^{\prime}}{\partial y}\right)^{2}+\left(\frac{\eta^{\prime}}{\sqrt{1+B^{2}}}+\alpha \sqrt{1+B^{2}} \frac{\partial \chi^{\prime}}{\partial x}\right)^{2}+\left[1-\alpha^{2}\left(1+B^{2}\right)\right]\left(\frac{\partial \chi^{\prime}}{\partial x}\right)^{2}\right)\right. \\
\left.+\int_{0}^{\tilde{q}^{\prime}}\left[G_{\alpha}\left(\tilde{q}_{0}+\xi\right)-G_{\alpha}\left(\tilde{q}_{0}\right)\right] d \xi\right\} d \boldsymbol{x} .
\end{aligned}
$$

This expression is seen to be positive definite if

$$
\left(U_{0}+\alpha\right) \frac{d \tilde{q}_{0}}{d y}<0 \quad \forall y \quad \text { and } \quad \alpha^{2}<\frac{1}{1+B^{2}}
$$

using $d G_{\alpha} / d \tilde{q}_{0}=\left[d G_{\alpha} / d y\right] /\left(d \tilde{q}_{0} / d y\right)=-\left(U_{0}+\alpha\right) /\left(d \tilde{q}_{0} / d y\right)$, where $U_{0} \equiv-d \psi_{0} / d y$. (Note that under the condition $(5.4 \mathrm{a}), G_{\alpha}(\cdot)$ is guaranteed to be single-valued.) Since $\mathcal{A}$ is an exact invariant of the nonlinear dynamics, it follows using standard methods that whenever $\alpha$ can be chosen such that $(5.4 \mathrm{a}, \mathrm{b})$ are satisfied, the basic state is nonlinearly stable. In particular, a normed stability theorem can be established $\dagger$, which holds for disturbances of arbitrarily large magnitude.

It was Arnol'd (1966) who first introduced this approach [building on earlier work of Fjørtoft (1950)] and applied it to the barotropic system. For that system, (5.4a) alone is a sufficient condition for nonlinear stability. The same result applies to the quasi-geostrophic system. Our stability theorem is thus seen to be the analogue of Arnol'd's theorem for the weak-wave model, with the additional condition (5.4b) arising from the model's gravity wave (unbalanced) dynamics.

Ripa (1983) followed the above methodology for the full shallow-water system, and showed that the second variation of the corresponding invariant $\mathcal{A}$ was positive definite if an $\alpha$ could be found such that (in terms of our present variables)

$$
\left(U_{0}+\alpha\right) \frac{d q_{0}}{d y}<0 \quad \forall y \quad \text { and } \quad\left(\alpha+U_{0}\right)^{2}<\frac{1+\eta_{0}}{1+B^{2}} \quad \forall y
$$

$\dagger$ Technically this requires that the left-hand side of $(5.4 \mathrm{a})$ be bounded away from zero and minus infinity. 
where $q_{0}=\left(b+\nabla^{2} \psi_{0}\right) /\left(1+\eta_{0}\right)$ is a scaled potential vorticity. Note that under the rescaling implicit in (3.7), $\left|\eta_{0}\right|=O(\epsilon) \ll 1$ so $q_{0} \approx b+\tilde{q}_{0}$ and the right-hand side of $(5.5 \mathrm{~b})$ reduces to the right-hand side of $(5.4 \mathrm{~b})$. Thus the stability theorem resulting from conservation of $\mathcal{A}$ in the weak-wave model is analogous to that in the full shallow-water model. The key difference is that the higher-order terms in the shallow-water form of $\mathcal{A}$ cannot be controlled (it appears that only the second variation of $\mathcal{A}$ can be shown to be positive definite), so condition (5.5) is only a statement of small-amplitude (linear) stability.

The fact that the weak-wave stability theorem is fully nonlinear means that it can be used to obtain rigorous upper bounds on the nonlinear saturation of instabilities in this model, following the method of Shepherd (1988). In Section 6 it is shown how this technique places rigorous bounds (within the context of this weak-wave model) on the emission of gravity waves from unstable parallel vortical flows.

\section{(b) Axisymmetric basic states}

We now consider disturbances to a steady axisymmetric basic state $\left\{\tilde{q}_{0}(r), \Delta_{0}(r), \eta_{0}(r)\right\}$ where $x=r \cos \theta, y=r \sin \theta$. In this case we proceed exactly as above, but using the angular momentum invariant (4.5) in place of the zonal momentum. Defining $\mathcal{P}$ according to (5.1) but with $\mathcal{M}$ replaced by $\mathcal{J}$, the extremal condition $\delta \mathcal{P}=0$ leads to

$$
C^{\prime}\left(\tilde{q}_{0}\right)=\psi_{0}+\alpha \frac{r^{2}}{2}, \quad \Delta_{0}=0, \quad \eta_{0}=b\left(1+B^{2}\right) \psi_{0}
$$

Introducing the function $G_{\alpha}(\cdot)$ defined by $G_{\alpha}\left(\tilde{q}_{0}(r)\right)=\psi_{0}(r)+\alpha r^{2} / 2$, it follows that $\mathcal{P}$ can be written

$$
\begin{aligned}
\mathcal{P}=\int\left\{\frac{1}{2}\left(\left|\nabla \psi^{\prime}\right|^{2}+\left|\nabla \chi^{\prime}\right|^{2}+\frac{\left(\eta^{\prime}\right)^{2}}{1+B^{2}}\right)-\psi_{0} \tilde{q}^{\prime}+\int_{\tilde{q}_{0}}^{\tilde{q}_{0}+\tilde{q}^{\prime}} G_{\alpha}(\xi) d \xi-\alpha \frac{r^{2}}{2} \tilde{q}^{\prime}+\alpha \eta^{\prime} \frac{\partial \chi^{\prime}}{\partial \theta}\right\} d \boldsymbol{x} \\
=\int\left\{\frac{1}{2}\left(\left|\nabla \psi^{\prime}\right|^{2}+\left(\frac{\partial \chi^{\prime}}{\partial r}\right)^{2}+\left(\frac{\eta^{\prime}}{\sqrt{1+B^{2}}}+\alpha \sqrt{1+B^{2}} \frac{\partial \chi^{\prime}}{\partial \theta}\right)^{2}+\left[\frac{1}{r^{2}}-\alpha^{2}\left(1+B^{2}\right)\right]\left(\frac{\partial \chi^{\prime}}{\partial \theta}\right)^{2}\right)\right. \\
\left.+\int_{0}^{\tilde{q}^{\prime}}\left[G_{\alpha}\left(\tilde{q}_{0}+\xi\right)-G_{\alpha}\left(\tilde{q}_{0}\right)\right] d \xi\right\} d \boldsymbol{x} .
\end{aligned}
$$


As with $\mathcal{A}, \mathcal{P}$ is an exact invariant of the nonlinear dynamics. The expression (5.7) is seen to be positive definite if

$$
\left(U_{0}+\alpha r\right) \frac{d \tilde{q}_{0}}{d r}>0 \quad \forall r \quad \text { and } \quad \alpha^{2}<\frac{1}{r^{2}\left(1+B^{2}\right)},
$$

where $U_{0} \equiv d \psi_{0} / d r$. However, it turns out that for physically realizable flows in an unbounded domain, $(5.8 \mathrm{a}, \mathrm{b})$ can never be satisfied! To see this, first note that taking $r \rightarrow \infty$ in (5.8b) implies that $\alpha=0$. Using (5.6c), $\tilde{q}_{0}=d^{2} \psi_{0} / d r^{2}+(1 / r) d \psi_{0} / d r-B^{2} \psi_{0}$. Therefore $(5.8 \mathrm{a})$ requires

$$
\begin{gathered}
0<\int_{0}^{\infty} \frac{d \psi_{0}}{d r} \frac{d \tilde{q}_{0}}{d r} d r=\int_{0}^{\infty}\left\{\frac{d \psi_{0}}{d r} \frac{d^{3} \psi_{0}}{d r^{3}}+\frac{d \psi_{0}}{d r} \frac{d}{d r}\left(\frac{1}{r} \frac{d \psi_{0}}{d r}\right)-B^{2}\left(\frac{d \psi_{0}}{d r}\right)^{2}\right\} d r \\
=-\int_{0}^{\infty}\left\{\left(\frac{d^{2} \psi_{0}}{d r^{2}}\right)^{2}+B^{2}\left(\frac{d \psi_{0}}{d r}\right)^{2}\right\} d r+\int_{0}^{\infty} \frac{d \psi_{0}}{d r} \frac{d}{d r}\left(\frac{1}{r} \frac{d \psi_{0}}{d r}\right) d r
\end{gathered}
$$

using the conditions $d U_{0} / d r \rightarrow 0$ as $r \rightarrow \infty$ and $U_{0} \rightarrow 0$ as $r \rightarrow 0$. But

$$
\begin{aligned}
\int_{0}^{\infty} \frac{d \psi_{0}}{d r} \frac{d}{d r}\left(\frac{1}{r}\right. & \left.\frac{d \psi_{0}}{d r}\right) d r=\int_{0}^{\infty}\left\{\frac{1}{r} \frac{d \psi_{0}}{d r} \frac{d^{2} \psi_{0}}{d r^{2}}-\frac{1}{r^{2}}\left(\frac{d \psi_{0}}{d r}\right)^{2}\right\} d r \\
& =-\int_{0}^{\infty} \frac{d \psi_{0}}{d r} \frac{d}{d r}\left(\frac{1}{r} \frac{d \psi_{0}}{d r}\right) d r-\int_{0}^{\infty} \frac{1}{r^{2}}\left(\frac{d \psi_{0}}{d r}\right)^{2} d r
\end{aligned}
$$

since $U_{0}=O(r)$ as $r \rightarrow 0$ for finite vorticity. Hence the second integral on the right-hand side of (5.9) is negative definite, which implies that (5.8) can never be satisfied.

In the barotropic or quasi-geostrophic case, (5.8a) alone is a sufficient condition for nonlinear stability, with no restriction on $\alpha$. In the shallow-water case, even a smallamplitude stability theorem cannot be obtained by this approach for unbounded domains (see Appendix). So we conclude that the weak-wave model captures the essential physics of the full shallow-water dynamics with regard to stability theorems of the Arnol'd type.

\section{Rigorous upper bounds on gravity-wave emission from unstable parallel shear flows}

Suppose we are given a parallel shear flow $\left\{\tilde{q}_{1}(y), \eta_{1}(y)\right\}$ which is known to be unstable. In general we expect the instability, even if it is vortical in nature, to excite gravity 
waves. For example, Ford (1994b) has studied shear-layer (Rayleigh-type) instability in the shallow-water equations, and has found the generation of gravity waves to be ubiquitous. An important question is whether there are any a priori upper bounds on the amplitude of such gravity-wave emission.

Such bounds can be obtained using our nonlinear stability theorem. Following Shepherd (1988), we may regard the initial unstable flow as a finite-amplitude disturbance to some nearby stable flow, and then invoke the nonlinear stability theorem. Since steady flows are necessarily balanced (with no gravity-wave component), any bound on the disturbance will also provide a bound on the gravity-wave activity. We provide an explicit demonstration of this by deriving a bound on the kinetic energy of the divergent flow,

$$
\mathcal{E}_{d} \equiv \int \frac{1}{2}|\nabla \chi|^{2} d x
$$

Let us assume, for the sake of simplicity, that the unstable flow is perturbed infinitesimally at $t=0$. (It would be straightforward to include the effect of a finite initial perturbation.) Now, introduce a basic state $\left\{\tilde{q}_{0}(y), \eta_{0}(y)\right\}$ that is stable by criteria (5.4). At $t=0$, the disturbance $\left\{\tilde{q}^{\prime}, \Delta^{\prime}, \eta^{\prime}\right\}$ relative to this basic state is given by $\eta^{\prime}(0) \approx \eta_{1}-\eta_{0}$, etc., and the invariant $\mathcal{A}$ is approximately given by

$$
\mathcal{A}(0)=\int\left\{\frac{1}{2}\left[\left(\frac{d\left(\psi_{1}-\psi_{0}\right)}{d y}\right)^{2}+\frac{\left(\eta_{1}-\eta_{0}\right)^{2}}{1+B^{2}}\right]+\int_{\tilde{q}_{0}}^{\tilde{q}_{1}}\left[G_{\alpha}(\xi)-G_{\alpha}\left(\tilde{q}_{0}\right)\right] d \xi\right\} d \boldsymbol{x} .
$$

For $t>0$, we note that $\chi=\chi^{\prime}$ (since the basic state has no divergent component); then from (6.1) and (5.3), together with the conservation of $\mathcal{A}$ in time, we obtain the inequality

$$
\mathcal{E}_{d}(t) \leq \frac{\mathcal{A}(t)}{1-\alpha^{2}\left(1+B^{2}\right)}=\frac{\mathcal{A}(0)}{1-\alpha^{2}\left(1+B^{2}\right)},
$$

into which (6.2) may be substituted. (Note that the factor $1-\alpha^{2}\left(1+B^{2}\right)$ is always positive, by virtue of $(5.4 \mathrm{~b})$.) In this way we obtain an a priori upper bound on the divergent kinetic energy $\mathcal{E}_{d}(t)$ for all time, which is a functional of the initial unstable flow and of the chosen stable basic state (including the choice of $\alpha$ ). This places a rigorous bound on the emission 
of gravity waves from the unstable flow. Since the basic state is arbitrary, one may try to minimize the bound over all possible choices of stable basic state (cf. Shepherd 1988). In the context of the present weak-wave model, this analysis has been performed by Nore (1994) for the Bickley jet and the $\tanh (y)$ shear layer.

For an initial flow that is marginally unstable, the above procedure yields a bound that is a function of the supercriticality — implying weak gravity-wave emission. Arguments from statistical mechanics (Warn 1986) would suggest, to the contrary, that once a steady flow goes unstable, its energy will be partitioned roughly equally into both vortical and gravity-wave components. The nonlinear stability theorem therefore provides definite limits on the extent of such "thermalization" in the weak-wave model (cf. Shepherd 1987).

\section{Linear eigenvalues and instability}

The shallow-water equations allow mixed vortical/gravity-wave instabilities that are filtered by the quasi-geostrophic equations. An important test of our weak-wave model is the extent to which it can capture such instabilities. As a preliminary step, we consider the instability properties of two simple basic flows.

\section{(a) Zonally symmetric basic state}

We first consider the case of a piecewise-constant zonally symmetric potential vorticity distribution with a single jump at $y=0$. As such a flow has no intrinsic length scale, we may take $B=1$ without loss of generality, and introduce the basic state

$$
\tilde{q}_{0}=\left\{\begin{array}{cc}
2 Q, & y>0 \\
0, & y<0
\end{array} \quad, \quad \psi_{0}=\left\{\begin{array}{cc}
-2 Q+Q \mathrm{e}^{-y}, & y>0 \\
-Q \mathrm{e}^{y}, & y<0
\end{array},\right.\right.
$$

with $\eta_{0}=\sqrt{2} \psi_{0}$. Again without loss of generality, we take $Q>0$. The velocity field associated with this basic state is given by $U_{0}(y)=Q \mathrm{e}^{-|y|}$, namely an eastward symmetric jet with a maximum at $y=0$.

Physically this problem consists of gravity waves in the upper and lower half-planes, coupled to a potential-vorticity disturbance at $y=0$. In each of the two half-planes we 
introduce a normal-mode form for $\psi^{\prime}$, proportional to $\mathrm{e}^{i(k x+\ell y-\omega t)}$, and insist on evanescence as $|y| \rightarrow \infty$. The frequency $\omega$ must of course be the same in the two half-planes; the same condition applies to the (real) zonal wavenumber $k$, by virtue of the kinematic condition that $v^{\prime}=\psi_{x}^{\prime}$ must be continuous across $y=0$. Away from $y=0$, we have $\tilde{q}^{\prime}=0$ and so $(3.7 \mathrm{~b}, \mathrm{c})$ - with $B=1$ - yield

$$
\omega^{2}=\frac{1}{2}\left(1+k^{2}+\ell^{2}\right)
$$

which is just the dispersion relation for inertia-gravity waves. We obtain a dynamical matching condition at $y=0$ by integrating the linearized potential vorticity equation $(3.7 \mathrm{a})$, namely $\tilde{q}_{t}^{\prime}+U_{0} \tilde{q}_{x}^{\prime}+\psi_{x}^{\prime} \tilde{q}_{0 y}=0$, across $y=0$. This yields $\left(\omega-U_{0}(0) k\right)[\ell]=-i k\left[\tilde{q}_{0}\right]$, where [.] denotes the jump across $y=0$. Evidently a jump in $\ell$ is required to balance the jump in $\tilde{q}_{0}$. Since (7.2) implies that $\ell^{2}$ must be the same on either side of $y=0$, this means that $\ell$ must change sign. Letting $\ell$ denote the value for $y>0$, the dynamical matching condition may be written

$$
(\omega-Q k) \ell=-i Q k
$$

Equations (7.2) and (7.3) define the linear disturbance problem. Note that $\ell_{i}>0$ for evanescent disturbances; it may be shown that this requires the intrinsic frequency $\hat{\omega} \equiv$ $\omega-Q k$ to have a negative real part. For quasi-geostrophic dynamics, the condition (7.2) is replaced by the condition $\ell^{2}=-k^{2}-1$, which when substituted into (7.3) leads to the single (stable) solution branch

$$
\omega_{Q G}=Q k-\frac{Q k}{\sqrt{1+k^{2}}} .
$$

Condition (5.4a) is satisfied (in the appropriate limiting sense at $y=0$ ) if $\alpha<-Q$, so the basic state (7.1) is provably stable by our criterion (5.4) for $Q<Q_{c} \equiv 1 / \sqrt{2}$. This stability condition has a simple physical interpretation. From (7.2), the phase speed of the gravity-wave mode is always greater than $1 / \sqrt{2}$, but approaches that value in the limit $k \rightarrow \infty$. From (7.4), the phase speed of the quasi-geostrophic mode is always less than $Q$ but approaches that value in the limit $k \rightarrow \infty$. So when $Q<Q_{c}$, the phase speeds of the 
two modes are always separate; but when $Q>Q_{c}$, they cross for sufficiently large $k$. Our stability condition (5.4) is therefore a condition for the absence of a mode crossing.

In terms of dimensional variables, the critical value corresponds to $[\tilde{q}]=2 f$. This lies outside the formal domain of validity of the weak-wave model, since it requires order-unity relative changes in the layer depth.

Combining (7.2) and (7.3) leads to the quartic equation

$$
2 \hat{\omega}^{4}+4 Q k \hat{\omega}^{3}+\left(\left[2 Q^{2}-1\right] k^{2}-1\right) \hat{\omega}^{2}+Q^{2} k^{2}=0
$$

We can analyze (7.5) asymptotically in the limits of small and large wavenumber $k$. Note that the coefficient of $\hat{\omega}^{2}$ changes character depending on the value of $Q$ : for $Q<Q_{c}$, it is always negative; for $Q>Q_{c}$, it changes sign at some value of $k$. This latter case corresponds, as anticipated, to a mode crossing, as shown below.

For $k \ll 1$, the method of dominant balance leads to the two stable solutions

$$
2 \hat{\omega}^{4} \sim \hat{\omega}^{2} \quad \Longrightarrow \quad \hat{\omega} \sim-\frac{1}{\sqrt{2}}, \quad \omega \sim-\frac{1}{\sqrt{2}}
$$

and

$$
\hat{\omega}^{2} \sim Q^{2} k^{2} \quad \Longrightarrow \quad \hat{\omega} \sim-Q k, \quad \omega \sim \frac{Q k^{3}}{2} .
$$

In each case there are two real roots but we have kept only the one with $\hat{\omega}<0$. The root (7.6) evidently corresponds to the small- $k$ limit of a pure gravity-wave mode (7.2), while the root (7.7) corresponds to the small- $k$ limit of the quasi-geostrophic mode (7.4). (The expression for $\omega$ in (7.7) obtains on expanding (7.5) in $k$.)

For $k \gg 1$, the method of dominant balance leads to the solutions

$$
2 \hat{\omega}^{4}+4 Q k \hat{\omega}^{3} \sim\left(1-2 Q^{2}\right) k^{2} \hat{\omega}^{2} \quad \Longrightarrow \quad \hat{\omega} \sim-Q k \pm \frac{k}{\sqrt{2}}, \quad \omega \sim \pm \frac{k}{\sqrt{2}}
$$

and

$$
\left(1-2 Q^{2}\right) k^{2} \hat{\omega}^{2} \sim Q^{2} k^{2} \quad \Longrightarrow \quad \hat{\omega} \sim \pm \frac{Q}{\sqrt{1-2 Q^{2}}}, \quad \omega \sim Q k
$$


There are two distinct cases here. When $Q<Q_{c}$, then only the negative root of (7.8) has $\hat{\omega}<0$; it is evidently the large- $k$ limit of a pure gravity-wave mode (7.2). In this case another stable solution is provided by the negative root of (7.9); it reduces to the large- $k$ limit of the quasi-geostrophic mode (7.4) for $Q \ll 1$, and generally has the same characteristic of the intrinsic frequency asymptoting to a constant. When $Q>Q_{c}$, on the other hand, (7.9) implies an unstable solution with $\hat{\omega}_{i} \neq 0$; in this case both roots of (7.8) have $\hat{\omega}<0$.

Therefore, for $Q<Q_{c}$, when our stability theorem predicts stability for this basic flow, we find that both quasi-geostrophic and gravity-wave neutral modes can be identified, and are connected smoothly (i.e. arise from the same dominant balance) in the small- $k$ and large- $k$ limits: there is no mode crossing. But for $Q>Q_{c}$, there is a dramatic change in the character of one of the solutions: the small- $k$ quasi-geostrophic mode metamorphoses into a gravity-wave mode in the large- $k$ limit, and an unstable solution appears.

Ford (1993) has studied the analogous basic state (with piecewise-constant $q$ rather than piecewise-constant $\tilde{q}$ ) in the context of the shallow-water equations, obtaining disturbance equations analogous to (7.2)-(7.3) but with the full basic-state height field entering the problem and with a Doppler shifting of the gravity-wave frequency. (Both of these effects are neglected in the weak-wave model.) Ford found a similar transition in the character of the neutral modes at a critical value of $Q$, corresponding to the critical value predicted by Ripa's stability criterion (5.5); for $Q$ larger than the critical value, he found mode crossing and instability. In terms of our non-dimensionalization, Ford's critical value of $Q$ (based on the jump in relative vorticity) is $3 /(4 \sqrt{2})$, which is somewhat less than our $Q_{c}$. Moreover, in the shallow-water case the growth rates are exponentially small for large $k$, whereas here they are of order unity. Both these discrepancies reflect the fact that the instability occurs outside the range of validity of the weak-wave model. 


\section{(b) Axisymmetric basic state}

We now consider the case of a piecewise-constant axisymmetric potential vorticity distribution with a single jump at $r=1$ :

$$
\tilde{q}_{0}=\left\{\begin{array}{ll}
Q, & r<1 \\
0, & r>1
\end{array} \quad, \quad B^{2} \psi_{0}=\left\{\begin{array}{rr}
-Q+B Q K_{1}(B) I_{0}(B r), & r<1 \\
-B Q I_{1}(B) K_{0}(B r), & r>1
\end{array},\right.\right.
$$

with $\eta_{0}=\left(1+B^{2}\right) b \psi_{0}$. Here $I_{m}$ and $K_{m}$ are modified Bessel functions of the first and second kind, of order $m$. The velocity field associated with this vortex is continuous in $r$, and has a maximum at $r=1$ given by $U_{0}(1)=Q K_{1}(B) I_{1}(B)$.

As in the zonally symmetric case, this problem consists of gravity waves inside and outside the vortex, coupled to a potential vorticity disturbance at $r=1$. Away from $r=1$, we introduce a normal-mode form for $\psi^{\prime}$, proportional to $f(r) \mathrm{e}^{i(m \theta-\omega t)}$ where $f(r)=I_{m}(\gamma r)$ for $r<1$ and $f(r)=I_{m}(\gamma) K_{m}(\gamma r) / K_{m}(\gamma)$ for $r>1$ (so $f(r)$ is continuous across $r=1)$. Taking $\tilde{q}^{\prime}=0$ for $r \neq 1,(3.7 \mathrm{~b}, \mathrm{c})$ yield the inertia-gravity wave dispersion relation

$$
\left(1+B^{2}\right) \omega^{2}=B^{2}-\gamma^{2} .
$$

As above, we obtain a dynamical matching condition at $r=1$ by integrating the linearized potential vorticity equation across $r=1$. After some algebra, this can be shown to reduce to

$$
\omega=Q m\left[K_{1}(B) I_{1}(B)-K_{m}(\gamma) I_{m}(\gamma)\right] .
$$

Equations (7.11) and (7.12) define the linear disturbance problem. The quasi-geostrophic problem consists of (7.12) alone with $\gamma$ replaced by $B$, which gives a single (stable) mode. In the limit $B \rightarrow 0$, this solution reduces to Kelvin's (1880) result $\omega=Q(m-1) / 2$.

The absence of a stability theorem in the axisymmetric case suggests the existence of mode crossing between the gravity-wave and quasi-geostrophic modes for all parameter values. That this is indeed the case can be seen as follows. In the limit $m \gg \max \{B, 1\}$, (7.12) suggests that $\omega=O(m)$; then (7.11) implies $\gamma \sim \pm i \sqrt{1+B^{2}} \omega$. Since $K_{m}(\gamma) I_{m}(\gamma)=$ $O(1 / \gamma)$ for $\gamma \gg 1$, the second term of (7.12) is much smaller than the first, which is 
consistent with the assumption that $\omega=O(m)$. Substituting this expression for $\gamma$ back into (7.12), an imaginary component to $\omega$ is implied: in particular,

$$
\omega_{r} \sim Q m K_{1}(B) I_{1}(B), \quad \omega_{i} \sim \pm \frac{\pi}{2} Q m\left[J_{m}\left(\sqrt{Q^{2} m^{2} K_{1}^{2}(B) I_{1}^{2}(B)\left(1+B^{2}\right)-B^{2}}\right)\right]^{2}
$$

where $J_{m}$ is the Bessel function of the first kind, of order $m$. We thus find instability for sufficiently large $m$, for all parameter values. In the limit of small $Q$ and large $m$, we may make the substitution $\operatorname{sech} \beta=Q K_{1}(B) I_{1}(B) \sqrt{1+B^{2}}$ in (7.13), and use the asymptotic approximation

$$
J_{m}(m \operatorname{sech} \beta) \sim \frac{\mathrm{e}^{m(\tanh \beta-\beta)}}{\sqrt{2 \pi m \tanh \beta}} .
$$

Together with the identity $\mathrm{e}^{2 \beta}=(1+\tanh \beta) /(1-\tanh \beta)$, this yields an approximate growth rate

$$
\omega_{i} \sim \frac{Q}{4} \frac{\mathrm{e}^{2 m \tanh \beta}}{\tanh \beta}\left(\frac{1-\tanh \beta}{1+\tanh \beta}\right)^{m}
$$

Now using the small- $Q$ expansion

$$
\tanh \beta=\sqrt{1-\operatorname{sech}^{2} \beta} \sim 1-\frac{1}{2} Q^{2} K_{1}^{2}(B) I_{1}^{2}(B)\left(1+B^{2}\right)+\ldots
$$

we obtain

$$
\omega_{i} \sim \frac{Q}{4} \mathrm{e}^{2 m}\left(\frac{1}{2} Q K_{1}(B) I_{1}(B)\left(1+B^{2}\right)^{1 / 2}\right)^{2 m}
$$

The derivation of this expression, valid for small $Q$ and large $m$, was provided by $\mathrm{R}$. Ford (personal communication, 1996). The Froude number based on the velocity at the vortex edge is $F=\left(1+B^{2}\right)^{1 / 2} U_{0}(1)$, namely $F=Q K_{1}(B) I_{1}(B)\left(1+B^{2}\right)^{1 / 2}$, so we see from (7.17) that the growth rate scales as $F^{2 m}$. This is in accord with the instability analysis of Ford (1994a) for the analogous basic state in the shallow-water equations (with piecewise-constant $q$ rather than piecewise-constant $\tilde{q}$ ); Ford obtained

$$
\omega_{i} \sim \frac{1}{4} \mathrm{e}^{2(m-1)}\left(\frac{F}{4}\right)^{2 m}
$$


in the limit of large $m$ and small $F$. The factor of $Q$ difference between (7.17) and (7.18) is accounted for by the different nondimensionalization of timescales, and the factor of $2^{2 m}$ difference by the fact that Ford's vortex-edge velocity is $1 / 2$ rather than 1 (so that there is a factor of two difference in the definition of $F$ ). The only remaining difference between (7.17) and (7.18) is a factor of $\mathrm{e}^{2}$, the origin of which is unclear.

When $B \ll 1$, then $K_{1}(B) I_{1}(B)\left(1+B^{2}\right)^{1 / 2} \rightarrow 1 / 2$ and the approximation $(7.15)$ is still valid for $Q<2$ (in the large- $m$ limit). In this case, the weak-wave model predicts a growth rate of $O(1)$ when $Q=O(1)$. This is very much larger than the shallow-water growth rate of $O\left(F^{2 m}\right)$ (see (7.18)) for $B \ll 1$ and $Q=O(1)$, and indicates that in this regime the weak-wave model is outside its range of validity. The problem is that although $B \ll 1$ for the vortex, the instability involves a gravity wave with a very long wavelength, whose frequency is not much larger than the rotation rate of the vortex. This illustrates the pitfalls of a formal scaling analysis based on a single length scale.

\section{Conclusion}

Atmospheric and oceanic flows can often be characterized as consisting of slow nonlin-

ear vortical motion evolving in the presence of fast but weak linear-wave motion. We seek a reduced dynamical model that can describe this situation, and that has the following features: quadratic energy; Hamiltonian structure; appropriate conservation laws; quasigeostrophic or barotropic vortical motion; fast waves; and non-trivial coupling between the vortical motion and the fast waves. In this paper we have derived such a "weak-wave" model in the context of the $f$-plane shallow-water equations, where the fast waves are inertia-gravity waves.

Although our model is appealing from the Hamiltonian perspective, it has at least two shortcomings. The first is that our method of approximation is not unique: if we had chosen different prognostic (dynamical) variables, we would have obtained a somewhat different system. This reflects the fact that our model is not based on a rigorous perturbation expansion. Against this we can only say that the problem of combining rigorous perturbation expansions with non-canonical Hamiltonian structure remains an open problem, which nobody has yet been able to solve. 
The second shortcoming of our model is that, for formal self-consistency, the fast waves must not be too weak. This might cast doubt on the utility of the model for studying mixed vortical/gravity-wave instabilities, and the breakdown of balanced dynamics. To determine whether the model is deficient in these respects, we have analyzed its stability and instability properties.

With regard to stability, the model possesses an Arnol'd-type stability theorem for zonally symmetric vortical basic flows, (5.4), that corresponds to the analogous theorem (5.5) for shallow-water dynamics, and that has the same physical interpretation: a basic flow is stable if its velocity is everywhere subcritical with respect to the gravity-wave phase speed. However, in our case the stability theorem is fully nonlinear. This fact permits the establishment of rigorous upper bounds on gravity-wave emission from unstable parallel shear flows. As is the case with shallow-water dynamics (see Appendix), an Arnol'd-type stability theorem cannot be obtained for axisymmetric vortical basic flows. This is in striking contrast with the quasi-geostrophic case — where any vortex with a monotonic potential-vorticity profile is nonlinearly stable — and reflects the destabilizing role of gravity waves in our model.

With regard to instability, we have examined the linear dynamics of disturbances to two piecewise-constant potential-vorticity distributions. In the case of the zonally symmetric basic state, corresponding to an exponential jet, the nature of the eigenvalue problem depends on the jet strength. When the jet is subcritical, the modes are everywhere neutral and can be clearly identified with quasi-geostrophic and gravity-wave modes. When the jet is supercritical, however, the eigenvalues collide (mode crossing) and instability occurs for sufficiently short zonal wavelengths. This behaviour corresponds qualitatively to that seen in the shallow-water system (Ford, 1993). In the case of the axisymmetric vortex, instability is found for all parameter values: gravity waves destabilize a vortex that would be stable under balanced dynamics. Once again, this is qualitatively consistent with the behaviour of the shallow-water system (Ford, 1994a). It is also consistent with the study of Broadbent \& Moore (1979), who found a destabilization of axisymmetric vortices by coupling to acoustic waves in the context of the two-dimensional compressible equations. 
The gravity-wave equation implied by (3.7) is

$$
\frac{\partial^{2} \Delta}{\partial t^{2}}+\left(\frac{B^{2}-\nabla^{2}}{1+B^{2}}\right) \Delta=-b \partial(\psi, \tilde{q})=-b \partial\left(\psi, \nabla^{2} \psi\right)+b^{2} \partial(\psi, \eta)
$$

Although the zeroth moment of the right-hand side of (8.1) clearly vanishes (for a compact vorticity distribution), the first moment does not appear to vanish. This is in contrast to the shallow-water system, which has vanishing zeroth and first moments (Ford et al. 1996), and suggests that our weak-wave model might overestimate the forcing of gravity waves by vortical motions (Ford, personal communication, 1996). However, the $\partial\left(\psi, \nabla^{2} \psi\right)$ term in (8.1) can be put into an explicitly quadrupolar form, which leaves only the $\partial(\psi, \eta)$ term as a dipolar source. For weak waves, $\eta$ is approximately given by $b\left(1+B^{2}\right) \psi$ (this is the quasi-geostrophic approximation), so this term may be expected to vanish to leading order in $\epsilon$. Thus it may well be that the gravity-wave source term in (8.1) has reasonable scaling properties in $\epsilon$. Further investigation of this point, through numerical simulations, would be of considerable interest.

Because the energy of our weak-wave model is quadratic, and the potential vorticity is a linear function of the other variables, this means that one can apply standard spectral arguments to deduce statistical mechanical equilibria and turbulent spectral regimes. In fact, Warn (1986) and Farge \& Sadourny (1989) have already done so. Although they were studying the shallow-water equations, they made the approximation of a quadratic energy and a linear potential vorticity in order to facilitate their analysis. It follows that their results apply directly to our weak-wave model.

Acknowledgements. This paper is the result of research begun at the 1993 Summer Study Program in Geophysical Fluid Dynamics at the Woods Hole Oceanographic Institution. We would thus like to thank Rick Salmon, the Director of the Program, for making this collaboration possible. We are also most grateful to Rupert Ford for many extremely helpful comments. The extension of the Lighthill theory to rotating shallow water was first performed by W. A. Norton (unpublished). TGS acknowledges support from the 
Natural Sciences and Engineering Research Council and the Atmospheric Environment Service of Canada.

\section{Appendix. On the non-existence of Arnol'd-stable circular vortices in unbounded shallow-water flow}

Using the Arnol'd-stability methodology followed in Section 5, Ripa (1987) derived a stability condition for axisymmetric basic flows in the context of the shallow-water equations. In terms of dimensional variables, Ripa (1987) showed that the small-amplitude (quadratic) approximation to the combined energy/angular-momentum/Casimir invariant $\mathcal{P}$ was positive definite if there existed a constant $\alpha$ such that

$$
(V+\alpha r) \frac{d Q}{d r}>0 \quad \forall r \quad \text { and } \quad(V+\alpha r)^{2}<g H \quad \forall r
$$

Here $V(r)$ is the basic-state azimuthal velocity, $Q(r)=(f+d V / d r+V / r) / H$ is the basicstate potential vorticity, and $H(r)$ is the basic-state total height, satisfying the gradientwind balance condition $g d H / d r=f V+V^{2} / r$. Conditions (A.1a,b) are clearly analogous to the stability conditions $(5.8 \mathrm{a}, \mathrm{b})$ for our weak-wave model, after the appropriate nondimensionalization.

However, it turns out that for physically realizable flows in an unbounded domain (A.1a,b), like $(5.8 \mathrm{a}, \mathrm{b})$, can never be satisfied. (Ford (1994a) has provided a heuristic argument to this effect for the special case of monotonic potential-vorticity profiles.) We confine our attention, as in Section $5 b$, to localized vortices with $d V / d r \rightarrow 0$ as $r \rightarrow \infty$. In that case $H(r)=o\left(r^{2}\right)$ as $r \rightarrow \infty$, and so the only way that (A.1b) can be satisfied in an unbounded domain is by taking $\alpha=0$. In that case the angular momentum invariant is not being used, and as Ripa (1987) argues, Arnol'd stability therefore cannot be proven. The argument involves an appeal to the result of Andrews (1984): if $\mathcal{P}$ consists only of the energy and a Casimir invariant, neither of which are altered by a spatial translation of the vortex, then the basic state cannot be a true extremum of $\mathcal{P}$ for arbitrary perturbations, and $\mathcal{P}$ cannot be sign definite. 
There are, however, some subtleties involved with the use of Andrews' theorem in an unbounded domain (Carnevale \& Shepherd 1990), and it is therefore desirable to establish the non-existence of Arnol'd-stable circular vortices by direct methods, as was done in Section $5 b$ for the weak-wave model. By definition, $r V(r)=\int_{0}^{r}(Q H-f) r^{\prime} d r^{\prime}$; this implies that $V=O(r)$ as $r \rightarrow 0$ (for finite vorticity) and that $d V / d r \rightarrow 0$ as $r \rightarrow \infty$ (for a localized vortex, with $Q H-f \rightarrow 0$ as $r \rightarrow \infty)$. Taking $\alpha=0$ as argued above, we now try to show that $($ A.1a,b) can never be satisfied. Noting that

$$
\frac{d Q}{d r}=\frac{1}{H}\left(\frac{d^{2} V}{d r^{2}}+\frac{1}{r} \frac{d V}{d r}-\frac{V}{r^{2}}\right)-\frac{Q}{g H}\left(f V+\frac{V^{2}}{r}\right),
$$

we obtain

$$
\begin{aligned}
\int_{0}^{\infty} H V \frac{d Q}{d r} d r & =-\int_{0}^{\infty}\left\{\left(\frac{d V}{d r}\right)^{2}+\frac{V^{2}}{2 r^{2}}+\frac{1}{g H}\left(f+\frac{d V}{d r}+\frac{V}{r}\right)\left(f V^{2}+\frac{V^{3}}{r}\right)\right\} d r \\
& =-\int_{0}^{\infty}\left\{\left(1-\frac{V^{2}}{4 g H}\right)\left(\frac{d V}{d r}\right)^{2}+\frac{V^{2}}{2 r^{2}}+\frac{V^{2}}{g H}\left(f+\frac{1}{2} \frac{d V}{d r}+\frac{V}{r}\right)^{2}\right\} d r
\end{aligned}
$$

Now, (A.1b) requires $V^{2}<g H$, in which case the right-hand side of (A.3) is negative definite; but (A.1a) requires the left-hand side of (A.3) to be positive definite. It follows that Ripa's stability conditions (A.1a,b) can never be satisfied in an unbounded domain. 


\section{References}

Andrews, D.G. 1984 On the existence of nonzonal flows satisfying sufficient conditions for stability. Geophys.Astrophys.Fluid Dyn., 28, 243-256.

Arnol'd, V.I. 1966 On an a priori estimate in the theory of hydrodynamical stability. Izv. Vyssh. Uchebn.Zaved.Matematika, 54, no.5, 3-5. (English transl.: Amer.Math.Soc.Transl., Series 2, 79, 267-269 (1969).)

Benjamin, T.B. 1984 Impulse, flow force and variational principles. IMA J.Appl.Math., 32, $3-68$.

Broadbent, E.G. \& Moore, D.W. 1979 Acoustic destabilization of vortices. Phil.Trans.Roy. Soc.Lond.A, 290, 353-371.

Carnevale, G.F. \& Shepherd, T.G. 1990 On the interpretation of Andrews' theorem. Geophys.Astrophys.Fluid Dyn., 51, 1-17.

Farge, M. \& Sadourny, R. 1989 Wave-vortex dynamics in rotating shallow water. J.Fluid Mech., 206, 433-462.

Fjørtoft, R. 1950 Application of integral theorems in deriving criteria of stability for laminar flows and for the baroclinic circular vortex. Geofys.Publ., 17, no.6, 1-52.

Ford, R. 1993 Gravity wave generation by vortical flows in a rotating frame. Ph.D. thesis, University of Cambridge, 289 pp.

Ford, R. 1994a The instability of an axisymmetric vortex with monotonic potential vorticity in rotating shallow water. J.Fluid Mech., 280, 303-334.

Ford, R. $1994 b$ Gravity wave radiation from vortex trains in rotating shallow water. J.Fluid Mech., 281, 81-118.

Ford, R., McIntyre, M. E. \& Norton, W. A. 1996 Balance and the slow quasi-manifold: some explicit results. J. Atmos. Sci. (to be submitted).

Kelvin, Lord 1880 On the oscillations of a columnar vortex. Phil.Mag., 10, 155-168.

Lorenz, E.N. 1960 Energy and numerical weather prediction. Tellus, 12, 364-373.

Lorenz, E.N. 1986 On the existence of a slow manifold. J.Atmos.Sci., 43, 1547-1557. 
Nore, C. 1994 A Hamiltonian weak-wave model for shallow water flow. In Geometrical methods in fluid dynamics (R. Salmon \& B. Ewing-Deremer, eds.), Woods Hole Oceanog. Inst. Tech. Report WHOI-94-12, pp.224-238.

Pedlosky, J. 1987 Geophysical Fluid Dynamics, 2nd ed. Springer-Verlag, 710 pp.

Ripa, P. 1983 General stability conditions for zonal flows in a one-layer model on the $\beta$-plane or the sphere. J.Fluid Mech., 126, 463-489.

Ripa, P. 1987 On the stability of elliptical vortex solutions of the shallow-water equations. J.Fluid Mech., 183, 343-363.

Sadourny, R. 1975 The dynamics of finite-difference models of the shallow water equations. J.Atmos.Sci., 32, 680-689.

Salmon, R. 1983 Practical use of Hamilton's principle. J.Fluid Mech., 132, 431-444.

Shepherd, T.G. 1987 Non-ergodicity of inviscid two-dimensional flow on a beta-plane and on the surface of a rotating sphere. J.Fluid Mech., 184, 289-302.

Shepherd, T.G. 1988 Rigorous bounds on the nonlinear saturation of instabilities to parallel shear flows. J.Fluid Mech., 196, 291-322.

Shepherd, T.G. 1990 Symmetries, conservation laws, and Hamiltonian structure in geophysical fluid dynamics. Adv.Geophys., 32, 287-338.

Shepherd, T.G. 1992 Arnol'd stability applied to fluid flow: successes and failures. In Nonlinear Phenomena in Atmospheric and Oceanic Sciences (G.F. Carnevale \& R.T. Pierrehumbert, eds.), 187-206. Springer-Verlag.

Shepherd, T.G. 1994 Applications of Hamiltonian theory to geophysical fluid dynamics. In Geometrical methods in fluid dynamics (R. Salmon \& B. Ewing-Deremer, eds.), Woods Hole Oceanog. Inst. Tech. Report WHOI-94-12, pp.113-152.

Warn, T. 1986 Statistical mechanical equilibria of the shallow water equations. Tellus, 38A, $1-11$.

Warn, T., Bokhove, O., Shepherd, T.G. \& Vallis, G.K. 1995 Rossby-number expansions, slaving principles, and balance dynamics. Quart.J.Roy.Meteor.Soc., 121, 723-739.

Yuan, L. \& Hamilton, K. 1994 Equilibrium dynamics in a forced-dissipative $f$-plane shallowwater system. J.Fluid Mech., 280, 369-394. 